\title{
ON LINEAR, SECOND ORDER DIFFERENTIAL EQUATIONS IN THE UNIT CIRCLE
}

\author{
BY \\ PHILIP HARTMAN AND AUREL WINTNER
}

1. Introduction. In the differential equation

$$
W^{\prime \prime}+p(z) W^{\prime}+q(z) W=0 \quad\left({ }^{\prime}=d / d z\right),
$$

let $z$ be a real or complex variable, $q(z)$ a continuous and $p(z)$ a continuously differentiable function on the domain under consideration. The function

$$
I(z)=q(z)-(1 / 4) p(z)-(1 / 2) p^{\prime}(z)
$$

is called the invariant of (1). If $W_{1}(z), W_{2}(z)$ are linearly independent solutions of (1) and if $u=W_{1} / W_{2}$, then

$$
2 I=\{u, z\},
$$

where $\{u, z\}$ is the Schwarzian parameter

$$
\{u, z\}=\left(u^{\prime \prime} / u^{\prime}\right)^{\prime}-(1 / 2)\left(u^{\prime \prime} / u^{\prime}\right)^{2} .
$$

The change of dependent variables $W \rightarrow w=W \exp \left((1 / 2) \int z p d z\right)$ transforms (1) into the normal form

$$
w^{\prime \prime}+I(z) w=0 .
$$

Hence, in considering zeros of solutions of (1), it can be assumed that (1) has the form (5). The term "solution" will always mean a non-trivial $(\not \equiv 0)$ solution.

This note will be concerned principally with solutions $w=w(z)$ of the differential equation (5) under the assumption that $I(z)$ is analytic on the circle $|z|<1$. (Unless the contrary is stated below, it will be supposed that $I(z)$ satisfies this assumption.)

The following terminology will be used: If no solution of a differential equation has two zeros (on a given $z$-set), then the differential equation will be said to be disconjugate (on that set) [11]. Similarly, if no solution has an infinite set of zeros, the differential equation will be called non-oscillatory. (In contrast to the situation on the real field, where Sturm's separation theorem is valid, it is possible that a solution of (5) can have a finite number of zeros on $|z|<1$ and that another solution has an infinite number of zeros there.)

2. Reduction to a real independent variable. The results on the zeros of solutions of (5) in the case that $z$ is a complex variable will be deduced from cases where $I(z)$ is a complex-valued function of a real variable (for example, $z=x+i y$ for fixed $y$ ). The transfer of these results from the real case will be possible because of the following "comparison" theorem (cf., e.g., [9, p. 319]):

Received by the editors April 14, 1954. 
$\left.{ }^{*}\right)$ Let $I(z)$ be a continuous, complex-valued function of a real variable $z$ on some interval. If

$$
v^{\prime \prime}+\Re(I(z)) v=0
$$

is disconjugate on the given interval, then (5) is disconjugate on that interval.

In this assertion, (6) can of course be replaced by any Sturm majorant, for example, by

$$
v^{\prime \prime}+|I(z)| v=0 .
$$

The comparison theorem $\left(^{*}\right)$ is a particular case of the following trivial fact: If $A_{1}$ and $A_{2}$ are self-adjoint (bounded or unbounded) operators in Hilbert space and $A_{1} \geqq$ const. $>0$, then $\lambda=0$ is not in the point spectrum of $A_{1}+i A_{2}$. Theorem (*) follows by choosing $A_{1} f$ to be the differential operator $f^{\prime \prime}+\Re I(z) f$ associated with boundary conditions $f=0$ at the end points of the interval and $A_{2} f$ to be $\Im(I(z)) f$.

The transformation rule

$$
\{u, Z\}=\{u, z\}(d z / d Z)^{2}+\{z, Z\}
$$

for the Schwarzian derivative under the change of (real or complex) independent variables $z \rightarrow Z$ supplies, by (3), the transformation rule for the invariant of (1) or (5). In particular, (8) reduces to

$$
\{u, Z\}=\{u, z\}(d z / d Z)^{2} \text { if } Z=(\alpha z+\beta) /(\gamma z+\delta),
$$

$\alpha \delta-\beta \gamma \neq 0$, since $w=\alpha z+\beta, \gamma z+\delta$ are linearly independent solutions of the equation $w^{\prime \prime}=0$ which has the invariant $I(z)=0$.

Assertion (*) immediately implies two results of Nehari [7], which state that if $u=u(z)$ is an analytic function on the unit circle $|z|<1$, then $u(z)$ is schlicht on $|z|<1$ whenever $I(z)$, defined by (3), satisfies either of the inequalities

$$
\begin{aligned}
& |I(z)| \leqq \pi^{2} / 4 \\
& |I(z)| \leqq 1 /\left(1-|z|^{2}\right)^{2}
\end{aligned}
$$

[7, p. 549 and p. 545]. In fact, $u(z)$ is schlicht on $|z|<1$ whenever (5) is disconjugate there. That either $\left(10_{1}\right)$ or $\left(10_{2}\right)$ implies that $(5)$ is disconjugate on $|z|<1$ can be deduced from $\left(^{*}\right)$ as follows:

Ad $\left(10_{1}\right)$. Suppose that some solution $w=w(z)$ of $(5)$ has two zeros in $|z|<1$, then the remark concerning (9) (in fact, the case $Z=e^{i \phi} z$ ) shows that there is no loss of generality in supposing that these zeros are on the same horizontal segment $z=x+i y$, with $y=$ const. and $x^{2}<1-y^{2}$. Since the length of the $x$-interval joining the two zeros is less than 2 , the inequality $\left(10_{1}\right)$ shows that no solution of $(7)$ (hence, by $\left({ }^{*}\right)$, no solution of $(5)$ ) can have two zeros on such an interval. 
Ad $\left(10_{2}\right)$. As verified by direct computation by Nehari $[7$, p. 547], the inequality $\left(1_{2}\right)$ for the invariant of $(5)$ is unchanged by conformal mappings $z \rightarrow Z$ of the unit circle $|z|<1$ onto $|Z|<1$. In fact, if

$$
d s=|d z| /\left(1-|z|^{2}\right)
$$

denotes the non-euclidean arc,length, which is invariant under the mapping $z \rightarrow Z$, then (9) can be written as

$$
\left(1-|Z|^{2}\right)^{2}|\{u, Z\}| d s=\left(1-|z|^{2}\right)^{2}|\{u, z\}| d s .
$$

Thus the invariance of $\left(10_{2}\right)$ follows from (3).

If the assertion concerning $\left(10_{2}\right)$ is false, then some solution $w=w(z)$ of (5) has at least two zeros on $|z|<1$. In view of the invariance of $\left(10_{2}\right)$, it can be supposed that these zeros are real. Either one of the following two equivalent arguments shows, by $\left({ }^{*}\right)$, that this leads to a contradiction. First, for real $z,(7)$ has the Sturm majorant

$$
d^{2} v / d x^{2}+v /\left(1-x^{2}\right)^{2}=0, \quad-1<x<1,
$$

which is disconjugate since it possesses the solution $v=\left(1-x^{2}\right)^{1 / 2}$ having no zeros on $-1<x<1$; cf. [6]. Second, the change of variables

$$
s=(1 / 2) \log (1+z) /(1-z)
$$

satisfying (11) for real $z=x$ transforms the invariant $|I(z)|$ of (7), according to (3) and (8), into the non-positive function $|I(z)|\left(1-|z|^{2}\right)^{2}-1$ for $z=z(s)$, $-\infty<s<\infty$. Hence, in the case $\left(10_{2}\right)$, (5) is disconjugate on $|z|<1$.

The constants $\pi^{2} / 4$ and 1 are the best possible in $\left(10_{1}\right)$ and $\left(10_{2}\right)$, respectively; $[7$, p. 549] and $[6$, p. 552].

3. A criterion for disconjugateness. The condition $\left(10_{2}\right)$ can be replaced by a somewhat different criterion:

(i) If $C$ is a circular arc in $|z|<1$ orthogonal to the boundary $|z|=1$, then the inequality

$$
\int_{C}\left(1-|z|^{2}\right)|I(z) d z| \leqq 2
$$

implies that (5) is disconjugate on $C$.

If $z=x$ in (7) is a real variable on some interval $a<x<b$ and $I(z)$ is continuous on this interval, then, according to [4], (7) is disconjugate on this interval if

$$
\int_{a}^{b}(b-x)(x-a)|I(x)| d x \leqq b-a
$$

(cf. [8] for a generalization to complex variables). Hence, if $C$ is the real interval $-1<x<1,(13)$ and $\left(^{*}\right)$ imply that no solution of $(5)$ has two zeros 
on the real axis. If $C$ is any circular arc orthogonal to $|z|=1$, there exists a transformation $z \rightarrow Z$ of the type in (9) of the circle $|z|<1$ onto $|Z|<1$ such that the image of $C$ is the real segment $-1<X<1$, where $Z=X+i Y$. It follows from (9) that no solution of (5) has two zeros on $C$ if

$$
\int_{-1}^{1}\left(1-|Z|^{2}\right)\left|I(z)(d z / d Z)^{2} d Z\right| \leqq 2, \quad Z=X .
$$

Note that (11) and (12) imply that

$$
\left(1-|Z|^{2}\right)|\{u, Z\} d Z|=\left(1-|z|^{2}\right)|\{u, z\} d z| \text {. }
$$

Consequently, the integrals in (13) and (15) are equal, and so (15) follows from (13). Thus (13) assures that no solution of (5) has two zeros on $C$.

4. Disconjugateness and $\mu(1)$. If $C$ is a line segment contained in $|z|<1$, a sufficient criterion for (7) (hence for (5)) to be disconjugate on $C$ is

$$
\int_{C}|I(z) d z| \leqq 4 / L, \quad \text { where } L \text { is the length of } C
$$

(Liapounoff; cf., e.g., [4]). If $C$ is a chord of $|z|=1$, this can be improved to

$$
\int_{C}\left(1-|z|^{2}\right)|I(z) d z| \leqq L
$$

by the criterion (14); see [4].

An inequality of Fejér and Riesz [3] states that

$$
\int_{C}|I(z) d z| \leqq(1 / 2) \mu(1)
$$

if $C$ is the real line segment $-1<x<1$,

$$
\mu(1)=\lim _{r \rightarrow 1} \mu(r),
$$

and

$$
\mu(r)=\int_{|z|=r}|I(z) d z| .
$$

According to a remark of Nehari [8, p. 695], (19) is valid if $C$ is any circular arc in $|z|<1$ orthogonal to $|z|=1$. Hence, the inequality

$$
\int_{C}\left(1-|z|^{2}\right)|I(z) d z| \leqq \int_{C}|I(z) d z|
$$

and (i) give the following:

(ii) The differential equation (5) is disconjugate on $|z|<1$ whenever 


$$
\mu(1) \leqq 4 .
$$

The weakened form $\mu(1) \leqq 2$ of this condition follows from Nehari's inequality (21) in [8] and his remark following it. The above use of the inequality (19) is similar to the procedure of Nehari.

The constant 4 in (22) is the ratio of the constants 2 in (13) and $1 / 2$ in (19). Although the inequalities (13) and (19) cannot be improved, it remains undecided whether or not (22) is the "best" possible.

Nehari's inequality leading to the weakened form $\mu(1) \leqq 2$ of (22) has the following consequence: The inequality

$$
\mu(r) \leqq 4 / L
$$

implies that no solution of (5) has two zeros $z=z_{1}, z_{2}$ in the circle $|z|<r$ satisfying $\left|z_{1}-z_{2}\right| \leqq L$.

5. Solutions satisfying $w(0)=0$. The inequality (23) can be considerably sharpened for $r$ near 1 in dealing with a particular solution of (5).

(iii) If $w=w(z)$ is a solution of (5) satisfying $w(0)=0$, then $w(z)$ has no zero different from $z=0$ in $|z|<1$ if

$$
\mu(r) \leqq 1 / 2 r(1-r) \text { for } 1 / 2 \leqq r<1
$$

In order to prove this, grant, for a moment, the fact that no solution of (7) (hence no solution of (5)) can have two zeros on a radius $z=t e^{i \phi}$, where $0 \leqq t<1$, if

$$
\int_{0}^{r} t^{2}\left|I\left(t e^{i \phi}\right)\right| d t \leqq r / 4(1-r) \text { for } 0<r<1 .
$$

The inequality of Fejér and Riesz implies the second of the inequalities

$$
\int_{0}^{r} t^{2}\left|I\left(t e^{i \phi}\right)\right| d t \leqq r^{2} \int_{-r}^{r}\left|I\left(t e^{i \phi}\right) d t\right| \leqq(1 / 2) r^{2} \mu(r)
$$

and so (24) implies (25). Thus, in order to prove (iii), it is sufficient to prove the statement concerning (25).

Let $q_{1}(s), q_{2}(s)$ be real-valued, continuous functions on $0<s<\infty$ such that

$$
q_{1}(s) \geqq 0 \quad \text { and } \quad \int^{\infty} q_{1}(s) d s<\infty .
$$

If the first of the differential equations

$$
d^{2} v / d s^{2}+q_{k}(s) v=0 \quad(k=1,2)
$$

is disconjugate on $0<s<\infty$ and if

$$
\int_{s}^{\infty}\left|q_{2}(s)\right| d s \leqq \int_{0}^{\infty} q_{1}(s) d s \quad(0<s<\infty),
$$


then $\left(26_{2}\right)$ is disconjugate on $0<s<\infty$ (cf. [5, p. 245] and [11]). The choice $q_{1}(s)=1 / 4 s^{2}$ (Kneser) gives the sufficient condition

$$
\int_{s}^{\infty}\left|q_{2}(s)\right| d s \leqq 1 / 4 s \quad(0<s<\infty)
$$

for $\left(26_{2}\right)$ to be disconjugate on $0<s<\infty$.

If $q(t)$ is continuous in the differential equation

$$
d^{2} v / d t^{2}+q(t) v=0 . \quad(0<t<1),
$$

the change of independent variables $s=(1-t) / t$ (which maps $0<t<1$ onto $\infty>s>0)$ alters the invariant $q(t)$ of $(29)$ to $q_{2}(s)=q(t)(d t / d s)^{2}$, by (9). Since (28) is transformed into

$$
\int_{0}^{t} t^{2}|q(t)| d t \leqq t / 4(1-t) \quad(0<t<1),
$$

the statement concerning (25) follows.

By using functions other than $q_{1}(s)=1 / 4 s^{2}$, for example,

$$
q_{1}(s)=\left(1 / 4 s^{2}\right)\left(1+1 / \log ^{2} s\right),
$$

it is possible to refine (24) somewhat. It is also possible to refine (iii) in the following direction: Let $0 \leqq \alpha<1$. There exists a constant $K=K$ (independent of $\alpha$ and $I(z))$ such that if

$$
\mu(r) \leqq K(1-\alpha)^{2} /(1-r) \text { for } 1 / 2 \leqq r<1,
$$

then no solution of (5) which has a zero in the circle $|z| \leqq \alpha$ has another zero on $|z|<1$.

6. The solutions in the case $\mu(1)<\infty$. If the condition (22) for (5) to be disconjugate on $|z|<1$ is weakened to

$$
\mu(1)<\infty,
$$

then, according to Nehari [8], (5) is non-oscillatory on $|z|<1$. Actually, (31) implies a great deal more about the solutions $w(z)$ of $(5)$ than the fact that $w(z)$ has only a finite number of zeros on $|z|<1$.

Let (31) hold. Then there exists a function $\psi(\theta)$ of bounded variation on $|\theta| \leqq \pi$ such that

$$
\psi(\theta)=\lim _{r \rightarrow 1} \int_{0}^{\theta}\left|I\left(r e^{i \phi}\right)\right| d \phi
$$

holds at every continuity point of $\psi(\theta)$.

The properties of the solutions $w(z)$ of (5) under the assumption (31) can be described as follows: 
$w(z)$ is uniformly continuous on $|z|<1$; in fact, the derivative $w^{\prime}(z)$ is bounded on $|z|<1$. In addition, the radial limits $w^{\prime}\left(e^{i \theta}\right)=\lim w^{\prime}\left(r e^{i \theta}\right)$, as $r \rightarrow 1$, exist for all $\theta$. The function $w^{\prime}(z)$ on $|z| \leqq 1$ (defined to be $w^{\prime}\left(e^{i \theta}\right)$ at $\left.z=e^{i \theta}\right)$ is continuous on $|z| \leqq 1$ except possibly at the points $e^{i \theta}$ where $\theta$ is a discontinuity point of $\psi(\theta)$. The point $z=e^{i \theta}$ is a continuity point of $w^{\prime}(z)$ if $w\left(e^{i \theta}\right)=0$. Finally, there exists one and only one solution $w=w(z)$ of $(5)$ for which $w$ and $w^{\prime}$ have preassigned radial limits $w\left(e^{i \theta}\right), w^{\prime}\left(e^{i \theta}\right)$ at a given point $z=e^{i \theta}$ of $|z|=1$.

In order to verify these properties, note that if, on a fixed radius, one has

$$
\int_{0}^{1}(1-t)\left|I\left(t e^{i \theta}\right)\right| d t<\infty
$$

then the radial limits $w\left(e^{i \theta}\right), w^{\prime}\left(e^{i \theta}\right)$ belonging to a solution $w(z)$ of $(5)$ exist ([1]; cf. [2, pp. 368-370] and [10, pp. 261-268]). Furthermore, there exists one and only one solution having preassigned radial limits $w\left(e^{i \theta}\right), w^{\prime}\left(e^{i \theta}\right)$ for a fixed $\theta$; cf. [10]. Clearly, (19) and (31) imply (33) for every $\theta$.

Every solution $w=w(z)$ of (5) satisfies

$$
w(z)=w(0)+z w^{\prime}(0)-\int_{0}^{z}(z-\zeta) I(\zeta) w(\zeta) d \zeta .
$$

Hence

$$
\left|w\left(r e^{i \theta}\right)\right| \leqq A+r \int_{0}^{r}\left|I\left(t e^{i \theta}\right) w\left(t e^{i \theta}\right)\right| d t \quad\left(A=|w(0)|+\left|w^{\prime}(0)\right|\right) .
$$

Consequently, a standard inequality gives

$$
\left|w\left(r e^{i \theta}\right)\right| \leqq A \exp r \int_{0}^{r}\left|I\left(t e^{i \theta}\right)\right| d t,
$$

and so, by the inequality of Fejér and Riesz,

$$
\left|w\left(r e^{i \theta}\right)\right| \leqq A \exp (1 / 2) r \mu(r) \leqq A \exp (1 / 2) \mu(1) .
$$

This proves that $w$ is bounded on $|z|<1$. By (34),

$$
w^{\prime}(z)=w^{\prime}(0)-\int_{0}^{z} I(\zeta) w(\zeta) d \zeta .
$$

Consequently,

$$
\left|w^{\prime}\left(r e^{i \theta}\right)\right| \leqq\left|w^{\prime}(0)\right|+\text { Const. } \int_{0}^{r}\left|I\left(t e^{i \theta}\right)\right| d t \leqq\left|w^{\prime}(0)\right|+\text { Const. } \mu(1),
$$

so that $w^{\prime}(z)$ is bounded on $|z|<1$.

The relation (35) shows that $w^{\prime}(z)$ is continuous at $z=e^{i \theta}$ if 


$$
\int_{\theta}^{\theta+h}\left|I\left(r e^{i \phi}\right) w\left(r e^{i \phi}\right)\right| d \phi \rightarrow 0, \quad \text { as } \quad(h, r) \rightarrow(0,1) .
$$

In view of the continuity of $w(z)$, this is the case when $\theta$ is a continuity point of $\psi(\theta)$ or when $w\left(e^{i \theta}\right)=0$. This completes the proof of the properties of $w(z)$ enumerated above.

It is clear that these properties imply that $w(z)$ has a finite number of zeros on $|z| \leqq 1$. For otherwise there is a point $z=e^{i \theta}$ which is a cluster point of zeros of $w(z)$. Then $w\left(e^{i \theta}\right)=0$ and so $w^{\prime}(z)$ is continuous at $z=e^{i \theta}$. Consequently $w^{\prime}\left(e^{i \theta}\right)=0$. But the only solution $w(z)$ belonging to the (radial) limits $w\left(e^{i \theta}\right)=0, w^{\prime}\left(e^{i \theta}\right)=0$ is the trivial solution $w(z) \equiv 0$.

7. An upper estimate for the number of zeros in $|z|<r$. The inequality (23) furnishes an upper estimate for the number $N(r)=N(r ; w(z))$ of zeros of a solution $w(z)$ in the circle $|z|<r(<1)$ :

(iv) Let there exist on $0<r<1$ a positive, continuously differentiable, non-decreasing function $\lambda=\lambda(r)$ satisfying

$$
\mu(r) \leqq \lambda(r)
$$

and

$$
d \lambda / d r=O\left(\lambda^{2}(r)\right), \quad \text { as } r \rightarrow 1 .
$$

Then

$$
N(r)=O\left(\int_{0}^{r} \lambda^{2}(r) d r\right)
$$

(iv) shows that if $z_{1}, z_{2}, \ldots$ are the zeros of a solution $w(z)$ of (5), then

$$
\sum\left(1-\left|z_{k}\right|\right)=\int(1-r) d N(r)<\infty
$$

is implied by

$$
\int^{1}(1-r) \lambda^{2}(r) d r<\infty .
$$

Simple examples seem to indicate that (38) can be improved to the corresponding relation in which $\lambda^{2}(r)$ is replaced by $\lambda(r)$. But this possibility will remain undecided.

In order to prove (iv), note that, according to the inequalities (23) and (36), the distance $L$ between any pair of zeros of a solution $w(z)$ on $|z| \leqq r$ satisfies $L \geqq 4 / \lambda(r)$. In the proof of (38), it can therefore be supposed that $\lambda(r) \rightarrow \infty$, as $r \rightarrow 1$.

If $r$ is sufficiently near 1 , the ring $r-2 / \lambda(r) \leqq|z| \leqq r$ can be divided into $2(2 \pi r) /(2 / \lambda(r))=2 \pi r \lambda(r)$ sets (curvilinear quadrilaterals) such that the dis- 
tance between any pair of points in any of the sets is less than $4 / \lambda(r)$. Consequently, the ring contains at most $2 \pi r \lambda(r)$ zeros of a solution $w=w(z)$. The width of the ring is $\Delta r=2 / \lambda(r)$. Hence, the number of zeros is at most $2 \pi r \lambda(r) \leqq$ Const. $\lambda^{2}(r) \Delta r$. On the other hand, the monotony of $\lambda$ implies

$$
\int^{r} \lambda^{2}(r) d r \geqq \sum \lambda^{2}(r-\Delta r) \Delta r,
$$

if the interval $(0, r)$ is divided into a finite number of pieces. Hence, it is clear that (38) follows if it is verified that

$$
\lambda(r)=O(\lambda(r-2 / \lambda(r)))
$$$$
\text { as } r \rightarrow 1 \text {. }
$$

But this is a consequence of (37), which implies that

$$
\log [\lambda(r) / \lambda(r-2 / \lambda(r))]=\int \lambda^{-1} d \lambda=O\left(\int \lambda d r\right)=O(1),
$$

as $r \rightarrow 1$, where the limits of integration are $r-2 / \lambda(r)$ and $r$.

\section{REFERENCES}

1. M. Bôcher, On regular singular points of linear differential equations of the second order whose coefficients are not necessarily analytic, Trans. Amer. Math. Soc. vol. 1 (1900) pp. 40-53.

2. O. Dunkel, Regular singular points of a system of homogeneous linear differential equations of the first order, Proceedings of the American Academy of Arts and Sciences vol. 38 (1902-03) pp 341-370.

3. L. Fejér and F. Riesz, Über einige funktionentheoretische Ungleichungen, Math. Zeit. vol. 11 (1921) pp. 305-314.

4. P. Hartman and A. Wintner, On an oscillation criterion of Liapounoff, Amer. J. Math. vol. 73 (1951) pp. 885-890.

5. E. Hille, Non-oscillation theorems, Trans. Amer. Math. Soc. vol. 64 (1948) pp. 234-252.

6. - Remarks on a paper by Zeev Nehari, Bull. Amer. Math. Soc. vol. 55 (1949) pp. $552-553$.

7. Z. Nehari, The Schwarzian derivative and schlicht functions, ibid. pp. 545-551.

8. - On the zeros of solutions of second order linear differential equations, Amer. J. Math. vol. 76 (1954) pp. 689-697.

9. C. T. Taam, Oscillation theorems, ibid. vol. 74 (1952) pp. 317-324. 251-272.

10. A. Wintner, Asymptotic integrations of the adiabatic oscillator, ibid. vol. 69 (1947) pp.

11. - On the non-existence of conjugate points, ibid. vol. 73 (1951) pp. 368-380.

The Johns Hopkins University, BALTIMORE, MD. 